\title{
ORIGIN OF COLOR GRADIENTS IN ELLIPTICAL GALAXIES
}

\author{
Naoyuki Tamura ${ }^{1}$, Chiaki Kobayashi ${ }^{2}$, Nobuo Arimoto $^{3}$, Tadayuki Kodama $^{2,4}$, \\ \& Kouji Ohta ${ }^{1}$ \\ ${ }^{1}$ Department of Astronomy, Faculty of Science, Kyoto University, Kyoto 606-8502, Japan \\ ${ }^{2}$ Department of Astronomy, School of Science, University of Tokyo, Tokyo 113-0033, Japan \\ ${ }^{3}$ Institute of Astronomy, School of Science, University of Tokyo, Mitaka, Tokyo 181-8588, \\ Japan \\ ${ }^{4}$ Physics Department, University of Durham, South Road, Durham, DH1 3LE, England \\ Aug. 3, 1999 \\ email: tamura@kusastro.kyoto-u.ac.jp
}

Received __; accepted _ 


\begin{abstract}
The origin of color gradients in elliptical galaxies is examined by comparing model gradients with those observed in the Hubble Deep Field. The models are constructed so as to reproduce color gradients in local elliptical galaxies either by a metallicity gradient or by an age gradient. By looking back a sequence of color gradient as a function of redshift, the age - metallicity degeneracy is solved. The observed color gradients in elliptical galaxies at $z=0.1-1.0$ agree excellently with those predicted by the metallicity gradient, while they deviates significantly from those predicted by the age gradient even at $z \sim 0.3$ and the deviation is getting larger with increasing redshift. This result does not depend on cosmological parameters and parameters for an evolutionary model of galaxy within a reasonable range. Thus our results clearly indicate that the origin of color gradients is not the age but the stellar metallicity.

Subject headings: galaxies: elliptical and lenticular, cD-galaxies: evolution - galaxies: formation
\end{abstract}




\section{INTRODUCTION}

Stellar populations in an elliptical galaxy are not uniform. Stars at a galaxy center are redder than those in the outer region and colors in a galaxy are progressively getting bluer with an increasing radius (e.g., Vader et al. 1988; Franx, Illingworth, \& Heckman 1989; Peletier et al. 1990a; Peletier, Valentijn, \& Jameson 1990b). Peletier et al. (1990a) made surface photometry in the $U_{-}, B$-, and $R$-bands for a sample of 39 nearby elliptical galaxies and examined their color gradients in $U-R$ and $B-R$. They found that typical color gradients $\Delta(U-R) / \Delta \log r$ and $\Delta(B-R) / \Delta \log r$ are $-0.20 \mathrm{mag} / \mathrm{dex}$ and $-0.09 \mathrm{mag} / \mathrm{dex}$, respectively, and demonstrated that a dispersion of the color gradients is small, i.e., only $0.02 \mathrm{mag} /$ dex in both colors. Since many of elliptical galaxies show radial gradients in

line strengths such as $\mathrm{Mg}_{2}, \mathrm{Fe}_{1}(5270 \AA)$ and $\mathrm{Fe}_{2}(5335 \AA)$ (e.g., Carollo, Danziger, \& Buson 1993; Davies, Sadler, \& Peletier 1993; Gonzalez 1993; Kobayashi \& Arimoto 1999), the color gradients have been naively assumed to originate from a metallicity gradient inside a galaxy.

However, such an interpretation for the origin of the color gradient is premature, because stellar populations of either higher metallicity or older age can make a galaxy redder. This problem, which is called the age-metallicity degeneracy, was first pointed out by Worthey, Trager, \& Faber (1996) and then discussed by Arimoto (1996). For example, the degeneracy makes it difficult to interpret the origin of the tight correlation between colors and magnitudes of elliptical galaxies; brighter elliptical galaxies tend to have redder colors. This correlation called color-magnitude (CM) relation can be excellently reproduced by a metallicity sequence with a galactic wind model based on a monolithic collapse scenario (e.g., Arimoto \& Yoshii 1987), where more massive elliptical galaxies should be enriched more in metals and thus become redder. However, Worthey et al. (1996) claimed that an age sequence of elliptical galaxies can equivalently reproduce the CM relation if brighter 
elliptical galaxies are older and thus redder. To break this degeneracy, Kodama \& Arimoto (1997) built up two model sequences (metallicity and age sequences) which are normalized to reproduce the CM relation of ellipticals in Coma cluster by using their evolutionary synthesis model. They compared the evolution of the model CM relation with the observed relations of ellipticals in distant clusters. The CM relations produced by a metallicity sequence agree with the observed relations out to $z \sim 1$, while those produced by an age sequence deviates from the observed ones significantly even at $z \sim 0.2-0.3$, showing the origin of the $\mathrm{CM}$ relation is primarily a metallicity variation with a galaxy mass. The $\mathrm{CM}$ relation can also be reproduced in a hierarchical galaxy formation scenario (Kauffmann \& Charlot 1998). Although a model of Kauffmann \& Charlot (1998) allows a more extended period of star formation in elliptical galaxies, it shows that the CM relation is produced by a metallicity variation. It is thus worth emphasizing that the interpretation of CM relation with a metallicity sequence is robust and independent of detailed assumptions on galaxy formation processes.

Recently, the origin of color and line strength gradients in elliptical galaxies has been discussed with elaborate models. Martinelli, Matteucci, \& Colafrancesco (1998) tried to reproduce the color gradients by assuming that a galactic wind blows later in the inner part of a galaxy due to a deeper potential well defined mainly by dark matter. Adopting a multi-zone model that takes into account gas dynamics, local star formation, and chemical evolution, Tantalo et al. (1998) reproduced radial gradients of colors and line strengths to some extent. Nevertheless, these detailed modelings are not fully successful; the inner part of a galaxy becomes too iron enriched due to an extended period of star formation there.

In this paper, adopting a much simpler approach without entering into details of physical processes of galaxy formation and evolution, we try to depict essential aspect of the origin of color gradients. Our approach is similar to that adopted by Kodama \& Arimoto 
(1997) for studying the origin of the CM relation. By using a population synthesis model, we first make two different model galaxies, both of which can reproduce a typical color gradient of elliptical galaxies at $z=0$, by changing either mean stellar metallicity or age, and let evolve them back in time. The evolution of color gradients thus predicted are then confronted with the observed ones in distant ellipticals extracted from the Hubble Deep Field North (HDF-N; Williams et al. 1996). As was demonstrated by Kodama \& Arimoto (1997), the best way to disentangle the age and metallicity effects on galaxy colors is to look back galaxies at high redshift.

It should be noted that dust extinction in elliptical galaxies may have some effects on the color gradients (Goudfrooij \& de Jong 1994; Wise \& Silva 1996). Witt, Thronson, \& Capuano (1992) calculated a radiative transfer within elliptical galaxies by assuming a diffuse distribution of dust and suggested that surface brightness profiles and color gradients could be well reproduced by dust effects. In fact, it has been believed that the IRAS detected FIR emission for about half of the elliptical galaxies observed. However, since many of the detections were made with a $\sim 3 \sigma$ threshold, a significant fraction of the previously claimed detections may be spurious. Only $12-17 \%$ of the observed elliptical galaxies are detected with a sufficiently high confidence level (Bregman et al. 1998). Therefore, in this paper, we have chosen to focus on age and metallicity effects only. Effects of dust extinction on the color gradients are still open and will be studied in our subsequent paper.

This paper is organized as follows. The model prescription is given in $\S 2$. The sample selection and data reduction of elliptical galaxies in the HDF-N are described in $\S 3$. The observed color gradients are compared with the models in $\S 4$. Discussion and conclusions are given in $\S 5$ and $\S 6$, respectively. The cosmological parameters adopted throughout this paper are $H_{0}=50 \mathrm{~km} \mathrm{~s}^{-1} \mathrm{Mpc}^{-1}, \Omega_{0}=0.2$ and $\Lambda=0$ unless otherwise noted. 


\section{MODELS}

The color gradients can be reproduced by either a metallicity gradient or an age gradient, but these effects are degenerate at $z=0$. We attempt to break up such degeneracy by confronting model color gradients with the observed ones of ellipticals at high redshifts in the HDF-N. We have built two sequences of evolutionary models under the alternative assumptions: 1) the color gradients originate from the metallicity gradient of old stellar populations, or 2) the color gradients arise from the age gradient of stars which have the same metallicity within the galaxy.

The metallicity sequence is constructed as follows: We assume that the star formation lasted progressively longer towards galaxy center; i.e., the galactic wind blew later in the inner region, so that the mean stellar metallicity becomes higher and the colors become redder. Even at the galaxy center, however, the star formation stopped at early times $\left(t_{\mathrm{gw}}=0.83 \mathrm{Gyr}\right)$ and the stellar ages are almost the same at everywhere within a galaxy; 15 Gyr at $z=0$ (see Tables 1 and 2). The assumption seems to be justified, because the duration of star forming activity is determined from the local dynamical potential (Larson 1974) and should be longer in the inner region. To build a metallicity gradient, however, this hypothesis is not unique; other hypotheses such as higher star formation efficiency, a larger yield (i.e., a flatter initial mass function (IMF) slope), or higher metallicity of infalling gas towards the galaxy center can also produce the metallicity gradient. However, such details are not essential in the present study, because so far as the mean stellar metallicity

increases in the inner part of a galaxy while stars are uniformly old, the resulting evolution of color gradient is essentially the same. We should stress this, because this paper aims for showing how color gradients of stellar populations behave as a function of lookback time, not for seeking the best model that can explain the observed color gradients of sampled galaxies in the HDF-N. The galactic wind is conventionally introduced to build up the 
metallicity gradient and it might be possible that such gradients can also be established if elliptical galaxies formed via hierarchical clustering.

The age sequence is constructed as follows: The star formation started earlier in the inner regions, but lasted equally long $\left(t_{\mathrm{gw}}=0.83 \mathrm{Gyr}\right)$ everywhere in a galaxy. The period chosen here is the same as that assumed for the center of metallicity sequence model. In this way, the mean stellar ages $t_{\text {age }}$ become older and colors redder in the inner regions, while the mean stellar metallicities are almost the same everywhere (see Tables 1 and 2). It is hard to comprehend the physical process to produce such age gradient, although it may occur if a series of young dwarf galaxies accrete onto a massive galaxy and are tidally disrupted at the outer region of a galaxy before falling into the center. However, it is not our aim anyway to construct a physically motivated age sequence model.

Although we do not discuss in this paper in detail, we have tried to build an alternative age sequence model in which star formation began everywhere 15 Gyr ago and lasted longer at the outer parts of the galaxy, keeping the same metallicity everywhere as that at the galaxy center. As a result, the mean stellar ages become progressively younger from the galaxy center towards the outer region. However, this model fails to reproduce the observed color gradients in nearby ellipticals. If old stellar populations are contained at the outer regions and if the mean metallicity is as rich as that at the center, there is no way by making their mean ages young to build up the color gradients as steep as the observed ones.

Both metallicity and age sequences are constructed to reproduce typical color gradients observed for nearby elliptical galaxies; $\Delta(B-R) / \Delta \log r=-0.09 \pm 0.02 \mathrm{mag} /$ dex (i.e., -0.07 and $-0.11 \mathrm{mag} / \mathrm{dex})$ and $B-R=1.633 \mathrm{mag}$ at $r=r_{\mathrm{e}} / 10\left(r_{\mathrm{e}}\right.$ refers to an effective radius) which is derived from the mean $B-R$ color at $r_{\mathrm{e}} / 2$ and $\Delta(B-R) / \Delta \log r=-0.09$ mag/dex (Peletier et al. 1990a). We also construct the models which reproduce the color gradients of $-0.09 \pm 0.04 \mathrm{mag} /$ dex (i.e., -0.05 and $-0.13 \mathrm{mag} / \mathrm{dex}$ ) within which the 
gradients of almost all the sample ellipticals in Peletier et al. (1990a) are included. Thus we have a set of four model sequences each for the age sequence and the metallicity sequence.

To calculate gradients of photo-chemical properties of a galaxy, we construct a galaxy model consisting of a 'shell' at each radius, and assume that each 'shell' evolves independently. The star formation rate (SFR) in a 'shell' is proportional to the gas fraction (Schmidt law) with a time scale of $0.1 \mathrm{Gyr}$ and a primordial gas is supplied to the shell with a rate of $\exp (-t / 0.1 \mathrm{Gyr})$. An infall of a primordial gas onto the shell may sound rather strange, since it is more likely that the inner shells suffer from the infall of enriched gas from the outer shells. However, a proper modeling of such effect is beyond our scope. Since the infall rate we employed in the present study is significantly high, the resulting evolutionary behavior of the color gradient remains almost the same even if we adopt the 'simple' model prescription instead of the infall model. For an IMF, a power-law mass spectrum with a slope of $x=1.10$ is assumed in the range of $0.05 M_{\odot} \leq M \leq 50 M_{\odot}$. We note that this IMF slope is the same as in Kodama et al. (1998), and was introduced to increase chemical yields to reproduce the reddest end of the Coma CM relation. The nucleosynthesis yields of SNe Ia and SNe II are taken from Tsujimoto et al. (1995). The chemical enrichment by SNe Ia is calculated with a metallicity dependent SN Ia rate, of which detail formulation is given by Kobayashi, Tsujimoto \& Nomoto (1999). The spectral evolution is calculated using a spectral synthesis database of Kodama \& Arimoto (1997). The adopted parameter values for galactic wind epoch $t_{\mathrm{gw}}$ and stellar age $t_{\mathrm{age}}$ at each radius for the four color gradients are listed in Table 1. The resulting photo-chemical properties of a galaxy at $z=0$ are summarized in Table 2, including gas abundances and colors at each radius. It should be noted that our models have an SN II-like abundance pattern, though the value $[\mathrm{Mg} / \mathrm{Fe}] \sim+0.4$ is slightly larger than that suggested by the observational estimates (Worthey, Faber \& Gonzalez 1992; Kobayashi \& Arimoto 1999); a larger [Mg/Fe] ratio is a result of the assumed flat $\operatorname{IMF}(x=1.10)$. 


\section{GALAXIES FOR COMPARISON}

\subsection{Sample Elliptical Galaxies}

To compare theoretical color gradients with those of elliptical galaxies at high redshifts, the archival data of the HDF-N (Williams et al. 1996) are used. Our sample galaxies are selected from those brighter than $I_{814, A B}=22 \mathrm{mag}$, in such a way that we can derive reliable surface brightness profiles and color gradients. All these galaxies have spectroscopic redshifts. Elliptical galaxies are identified by using a bulge-to-total $(B / T)$ luminosity ratio derived by Marleau \& Simard (1998) who obtained the $B / T$ ratios in the $I_{814}$-band of HDF galaxies brighter than $I_{814}=26$ mag by decomposing quantitatively the surface brightness profile into the bulge and the disk components. We define galaxies with $B / T>0.5$ as ellipticals according to Marleau \& Simard (1998). Our resulting sample consists of ten elliptical galaxies with redshifts spanning from $z=0.089$ to 1.015 as listed in Table 3. It is important to note here that our sample selection does not rely on any color information. Franceschini et al. (1998) also made a sample of elliptical galaxies in the HDF using surface brightness profiles. Their selection, however, is not based on the bulge - disk decomposition. Consequently, within $I_{814, A B}<22 \mathrm{mag}$, our sample is smaller than that of Franceschini et al. (1998), except for the galaxy 4-241.1 at $z=0.318$. The Franceschini et al's sample includes several galaxies having the $B / T$ ratio smaller than 0.5 , which are presumably disk dominant galaxies and thus are not included in our sample (see also Kodama, Bower \& Bell 1999). It should be noted that $B / T>0.5$ in the $I_{814}$-band may be slightly loose to isolate elliptical galaxies. $B / T>0.5$ in the $B$-band roughly corresponds to local early-type galaxies (e.g., Simien \& de Vaucouleurs 1986). By using bulge and disk models of Kodama et al. (1999), we found that the $I_{814}$-band $B / T$ ratios of ellipticals are larger than $\sim 0.7$ at $z=0$ and change little from $z=0$ to 1 in the observer's frame. Thus our sample may include galaxies as late as Sa, though they must be minority because most of the $B / T$ 
ratios of our sample galaxies are larger than 0.7 (Table 3).

We measured $V_{606}-I_{814}$ colors of our sample galaxies and present them in Table 3 and Figure 1. These colors are obtained for a $10 \mathrm{kpc}$ aperture. Seven of the sample galaxies have red colors consistent with those for ellipticals with passive evolution as shown in Figure 1 (hereafter we call these galaxies 'red ellipticals'), and the other three galaxies (4-241.1, 2-251.0, and 4-928.0) have blue colors (see also Figure 2; hereafter referred to as 'blue ellipticals' for convenience). Azimuthally averaged surface brightness profiles of the sample ellipticals in the $I_{814}$-band are shown in Figure 2. The average is taken along an ellipse fitted to an isophote of the $I_{814}$-band image. These profiles are well represented by an $r^{1 / 4}$ law for both the red ellipticals and the blue ellipticals. Effective radii $\left(r_{\mathrm{e}}\right)$ of the sample ellipticals are obtained by the $r^{1 / 4}$ fitting and results are shown in Table 3 . The fitting is done by removing data points in the inner and outer regions, since they are unreliable due to an effect of a point spread function (PSF) and low signal-to-noise ratios (S/Ns), respectively. The obtained effective radii range from $r_{e}=1.4$ to $7.9 \mathrm{kpc}$, which are typical for nearby giant ellipticals (e.g., Bender, Burstein, \& Faber 1992).

\subsection{Color Gradients}

To examine color gradients of our sample galaxies, the images in $\mathrm{F} 606 \mathrm{~W}\left(V_{606}\right)$ - and F814W $\left(I_{814}\right)$-bands were used, because $\mathrm{S} / \mathrm{Ns}_{\text {s }}$ of the images are better in these bands than in the other bands (F300W and F450W). First, the sky value around each object was determined by using the "phot" task in the IRAF apphot package ("mode" in an annulus with an inner radius of mostly $3^{\prime \prime} \sim 4^{\prime \prime}$ and a width of $0 . " 4$ is adopted) and was subtracted. Next, the angular resolutions of the blue images and the red images were adjusted and a $V_{606}-I_{814}$ color map of each galaxy was made. Figure 3 shows the resulting color maps. Most of the color maps for the red ellipticals show symmetric structure and ordinary color 
gradient. In two cases (2-456.0 and 2-121.0), slight asymmetry in the color distribution is seen. This does not seem to be caused by a misalignment of the images. For the blue ellipticals, variety of the color distribution is seen, which we will discuss in $§ 5.1$. Next, a $V_{606}-I_{814}$ color profile is derived with the azimuthally averaged radial profiles as a function of semi-major radius from the galaxy center. Resulting color profiles are shown in Figures 4 and 5 for the red ellipticals and in Figure 7 for the blue ellipticals. It should be noted that in plotting observed color profiles, a zero-point of the observed color for each galaxy is shifted to be compared with those by the models. The amounts of these shifts are given in the last column of Table 3. Most of the shifts are less than 0.2 mag; for intrinsically luminous objects the colors tend to be shifted to bluer colors and vice versa. Thus the color differences are presumably caused by an effect of the color-magnitude relation of elliptical galaxies. The zero-point offsets could also come from photometric errors in HST data (e.g., Holtzman et al. 1995; Ellis et al. 1997; Kodama et al. 1998) and from uncertainty of the population synthesis model. Needless to say, zero-point offsets do not affect the color gradients. An error bar attached to each data point in the color profile includes an photometric error, a local sky subtraction error, and a dispersion of colors along each elliptical isophote. The data points of 2-456.0 and 2-121.0 have relatively large error bars in the middle region of the profiles; these objects have asymmetric color distributions which results in the relatively large dispersions of colors in the regions.

Finally, slopes of the color profiles $\Delta\left(V_{606}-I_{814}\right) / \Delta \log \left(r / r_{\mathrm{e}}\right)$ of the sample galaxies are derived by applying a least square fit to the color profiles. This fit was done after removing the unreliable data points in the outer region $\left(r>r_{\mathrm{e}}\right)$ and in the innermost region. The removed data points in the inner most regions are shown by crosses in the color profiles (Figures 4 and 5); they clearly deviate from the $r^{1 / 4}$ fits presumably due to the effect of a PSF as seen in Figure 2. The slopes of the color profiles for the blue ellipticals presented in Figure 7 are not derived. 


\section{ORIGIN OF COLOR GRADIENTS}

Once the spectra are calculated at various radii of a model galaxy in such a way that they reproduce the color gradient $\Delta(B-R) / \Delta \log \left(r / r_{\mathrm{e}}\right)=-0.09 \mathrm{mag} / \mathrm{dex}$ at $z=0$, the model gradient in any colors at any redshifts can be predicted to compare with the observed gradients. The model color gradients at the redshift of each sample elliptical galaxy are over-plotted in each panel of Figures 4 and 5 for the age gradient and the metallicity gradient models, respectively. Solid and dotted lines in each panel show the gradients in $V_{606}-I_{814}$ color in the observer's frame corresponding to the gradients of $-0.09 \pm 0.02$ $\mathrm{mag} / \mathrm{dex}$ and $-0.09 \pm 0.04 \mathrm{mag} / \mathrm{dex}$ in the $B-R$ color at $z=0$, respectively.

It is clearly shown that the model gradient made by the age gradient begins to deviate from the observed ones at a redshift of $z \sim 0.3$ and the deviation is getting worse as a redshift increases (Figure 4). On the contrary, the model gradient made by the metallicity gradient agrees well with the observed color gradients within the effective radius at any redshift from $z \sim 0.1$ to $\sim 1$ (Figure 5). Considering that the model gradients are calibrated only at $z=0$, we insist that the agreement between the model and the observed gradients is excellent. Such agreement is more clearly seen in Figure 6 which shows both the observed color gradients and the model gradients as a function of redshift. Solid and dotted lines show the evolution of model color gradients in $V_{606}-I_{814}$ in the observer's frame for the metallicity gradient and the age gradient, respectively. These model gradients are calculated in a region of $-1 \leq \log r / r_{\mathrm{e}} \leq 0$ for the metallicity gradient and for the age gradient at $z<0.5$, and $-1 \leq \log r / r_{\mathrm{e}} \leq-0.5$ for the age gradient at $z>0.5$. Dots show the observed gradients of the sample galaxies within $r_{\mathrm{e}}$ calculated by a least square fitting to the data points shown as filled circles in Figures 4 and 5. Error bars indicate the fitting errors of $1 \sigma$.

\section{DISCUSSION}




\subsection{Blue Galaxies in Our Sample}

Since our sample is selected independently of galaxy colors, relatively blue elliptical-like galaxies are included in our sample (3/10). Among these three, 4-241.1 $(z=0.318)$ has a nearly flat color profile with a slightly bluer color in the inner part (Figure 3 and the top panel of Figure 7). The spectrum of this galaxy (from the Hawaii Active Catalog; Cowie 1997) shows Balmer absorption lines shortward of $4000 \AA$ in the rest frame as well as emission lines such as $[\mathrm{OII}] \lambda 3727, \mathrm{H} \beta,[\mathrm{OIII}] \lambda 4959, \lambda 5007$, and $\mathrm{H} \alpha$. Thus this galaxy is expected to have some young stellar populations. The galaxy $2-251.0(z=0.960)$ has a color gradient in the opposite sense to those of ordinary ellipticals; i.e., the inner region of the galaxy is bluer than the outer region (Figure 3 and the middle panel of Figure 7). Since this galaxy has been suggested as an AGN (Franceschini et al. 1998), this opposite gradient is probably caused by AGN and/or associating starburst activity in the central part of the galaxy. Although both galaxies seem to have the younger ages, our model by the age gradient cannot reproduce their color gradients. Accordingly, neither the metallicity gradient nor the age gradient can explain their observed color gradients. These galaxies presumably have on-going star formation (or post-starburst) in the inner part of the galaxies, which may be caused by a galaxy interaction as expected from their rather disturbed morphology. Our model cannot apply to these cases. The other galaxy 4-928.0 $(z=1.015)$ has a rather steep color gradient as seen in Figure 3 and the bottom panel of Figure 7. The observed color profile of the galaxy is shifted by 0.4 mag to be compared with the model color gradients (solid and dotted lines). The observed color gradient of this galaxy may be explained by the age gradient if we tune ages of stellar populations in the model galaxy. However, the mean age is set to be 0.2 Gyr at $r_{\mathrm{e}}$ and 9 Gyr at the innermost point. Such a large age at the center cannot be realized at this redshift $(z=1.015)$ unless a different set of cosmological parameters is adopted. Finally, note that colors of these galaxies are not consistent with those of passively evolving ellipticals formed at high $z$. 
Much larger sample is needed in future works to find whether the population of the blue galaxies is really the minority in the whole population of elliptical galaxies.

\section{2. $\quad$ Gradients of Metal Absorption Line Strengths}

Kobayashi \& Arimoto (1999) showed that central velocity dispersions of elliptical galaxies correlate well with their mean metallicities. They found, however, that this correlation has a significant scatter. They also showed that $\mathrm{Mg}_{2}$ gradients do not correlate with any physical parameters including a galaxy mass. Such a large dispersion and an absence of the correlation are contrary to what monolithic collapse simulations predicted. As the common origin for the large dispersion and the absence of the correlation, they suggested the following possibilities: (1) a significant age spread among elliptical galaxies, (2) age gradients inside elliptical galaxies, (3) dust obscuration, (4) effects of merging on metallicity gradients inside elliptical galaxies, and (5) intrinsic scatter which elliptical galaxies have had since they formed. Since we confirm in this paper that the color gradients are caused by the metallicity gradient, we can reject the possibility (2).

There seems to be a problem that color gradients in elliptical galaxies do not correlate with their $\mathrm{Mg}_{2}$ gradients, though only a small number of elliptical galaxies are examined both for color gradient and $\mathrm{Mg}_{2}$ gradient (Peletier 1989). However, the absence of this correlation may be a spurious result caused by a different way to derive the gradients between the color and $\mathrm{Mg}_{2}$ gradients. Some effects of dust extinction and/or, possibly, observational errors on both gradients might suppress the true correlation. In any case, this problem will be investigated in detail in our forthcoming paper.

Worthey et al. (1992) suggested that the color-magnitude relation of elliptical galaxies may originate from the fact that more luminous ellipticals tend to contain more $\mathrm{Mg}$ 
relative to Fe. One can claim that it could be possible that the same holds for the internal gradients. However, Kobayashi \& Arimoto (1999) have recently carried out a careful study of line strength gradients of 80 elliptical galaxies having the best quality of spectra but find no evidence supporting for the $[\mathrm{Mg} / \mathrm{Fe}]$ gradient. Therefore, we conclude that the color gradients are not originating from the gradient of the $[\mathrm{Mg} / \mathrm{Fe}]$ ratio.

\section{CONCLUSIONS}

The origin of color gradients in elliptical galaxies is examined. A typical color gradient of nearby elliptical galaxies is reproduced by two alternative model sequences, metallicity gradient and age gradient. This age-metallicity degeneracy is broken by looking back the evolution of color gradient towards high redshifts; the predicted color gradients at high redshifts are compared with the observed color gradients of ten elliptical galaxies in the HDF-N out to $z \sim 1$. We find that the observed color gradients of the seven red galaxies, whose colors are consistent with those of passively evolving galaxies, are in excellent agreement with the metallicity gradient at any redshift. These conclusions are independent of cosmological parameters and parameters for an evolutionary model of galaxy (IMF and SFR) within a reasonable range. Thus we conclude that the primary origin for the color gradient in elliptical galaxies is the metallicity gradient in the old stellar populations.

This work was financially supported in part by Grant-in-Aids for the Scientific Research (No. 0940311 and 09740173) by the Japanese Ministry of Education, Culture, Sports and

Science. CK and TK thank Research Fellowships of the Japan Society for the Promotion of Science (JSPS) for Young Scientists. 


\section{REFERENCES}

Abraham, R. G., Ellis, R. S., Fabian, A. C., Tanvir, N. R., \& Glazebrook, K. 1999, MNRAS, 303,641

Arimoto, N. 1996, in From Stars to Galaxies, eds. C. Leitherer, U. Fritze-v. Alvensleben, \& J. Huchra, ASP Conf. Ser. 98, 287

Arimoto, N., \& Yoshii, Y. 1987, A\&A, 173, 23

Bender, R., Burstein, D., \& Faber, S. M. 1992, ApJ, 399, 462

Bouwens, R., Broadhurst, T., \& Silk, J. 1998, ApJ, 506, 557

Bregman, J. N., Snider, B. A., Grego, R., \& Cox, C. V. 1998, ApJ, 499, 670

Carollo, C. M., Danziger, I. J., Buson, L. 1993, MNRAS, 265, 553

Cohen, J. G., Cowie, L. L., Hogg, D. W., Songaila, A., Blanford, R., Hu, E. M., \& Shopbell, P. 1996, ApJL, 471, L5

Cowie, A. S. 1997, unpublished catalog of HDF redshifts (Hawaii Active Catalog)

Davies, R. L., Sadler, E. M., \& Peletier, R. F. 1993, MNRAS, 262, 650

Ellis, R. S., Smail, I., Dressler, A., Couch, W. J., Oemler, A. Jr., Butcher, H., \& Sharples, R. M. 1997, ApJ, 483, 582

Franceschini, A., Silva, L., Fasano, G., Granato, G. L., Bressan, A., Arnouts, S., \& Danase, L. 1998, ApJ, 506, 600

Franx, M., Illingworth, G., \& Heckman, T. 1989, AJ, 98, 538

Gonzalez, J. J. 1993, PhD thesis, Univ. of California 
Goudfrooij, P. \& de Jong, T. 1994, A\&A, 298, 784

Holtzman, J. A., Burrows, C. J., Casertano, S., Hester, J. J., Trauger, J. T., Watson, A. M., \& Worthey, G. 1995, PASP, 107, 1065

Kauffmann, G., \& Charlot, S. 1998, MNRAS, 294, 705

Kobayashi, C., \& Arimoto, N. 1999, ApJ, in press (astro-ph/9907091)

Kobayashi, C., Tsujimoto, T., \& Nomoto, K. 1999, ApJ, submitted (astro-ph/9908005)

Kodama, T., \& Arimoto, N., 1997, A\&A, 320, 41

Kodama, T., Arimoto, N., Barger, A. J., \& Aragón-Salamanca, A. 1998, A\&A, 334, 99

Kodama, T., Bower, R. G., \& Bell, E. F. 1999, MNRAS, 306, 561

Larson, R. B. 1974, MNRAS, 169, 229

Marleau, F. R., \& Simard, L. 1998, ApJ, 507, 585

Martinelli, A., Matteucci, F., \& Colafrancesco, S. 1998, MNRAS, 298, 42

Peletier, R. F. 1989, PhD thesis, Univ. of Groningen

Peletier, R.F., Davies, R.L., Illingworth, G.D., Davis, L.E., \& Cawson M. 1990a, AJ, 100, 1091

Peletier, R. F., Valentijn, E. A., \& Jameson, R. F. 1990b, A\&A, 233, 62

Simien, F., \& de Vaucouleurs, G. 1986, ApJ, 302, 564

Tantalo, R., Chiosi, C., Bressan, A., Marigo, P., \& Portinari, L. 1998, A\&A, 333, 419

Tsujimoto, T., Nomoto, K., Yoshii, Y., Hashimoto, M., Yanagida, S., \& Thielemann, F.-K. 1995, MNRAS, 277, 945 
Vader, J. P., Vigroux, L., Lachiéze-Rey, M. \& Souviron, J. 1988, A\&A, 203, 217

Williams, R. E. et al. 1996, AJ, 112, 1335

Wise, M., \& Silva, D. R. 1996, ApJ, 461, 155

Witt, A. N., Thronson, H. A. Jr., \& Capuano, J. M. 1992, ApJ, 393, 611

Worthey, G., Faber, S. M., \& Gonzalez, J. J. 1992, ApJ, 398, 69

Worthey, G., Trager, S. C., \& Faber, S. M. 1996, in Fresh Views of Elliptical Galaxies, eds. A. Buzzoni, A. Renzini, A. Serrano, ASP Conf, Ser. Vol. 86, p. 203 
Table 1. Input Parameters

\begin{tabular}{|c|c|c|c|c|c|c|c|c|c|}
\hline $\log r / r_{\mathrm{e}}$ & -1 & -0.875 & -0.75 & -0.625 & -0.5 & -0.375 & -0.25 & -0.125 & 0 \\
\hline \multicolumn{10}{|c|}{ Metallicity Sequence $\left(t_{\text {age }}=15\right.$ Gyr $)$} \\
\hline$\Delta(B-R) / \Delta \log r$ & & & & & $t_{\mathrm{gw}}[\mathrm{Gyr}]$ & & & & \\
\hline-0.05 & 0.83 & 0.74 & 0.64 & 0.58 & 0.53 & 0.49 & 0.45 & 0.42 & 0.39 \\
\hline-0.07 & 0.83 & 0.69 & 0.59 & 0.52 & 0.47 & 0.42 & 0.38 & 0.35 & 0.32 \\
\hline-0.11 & 0.83 & 0.63 & 0.52 & 0.43 & 0.37 & 0.32 & 0.29 & 0.26 & 0.23 \\
\hline-0.13 & 0.83 & 0.60 & 0.48 & 0.39 & 0.33 & 0.29 & 0.26 & 0.22 & 0.20 \\
\hline \multicolumn{10}{|c|}{ Age Sequence $\left(t_{\mathrm{gw}}=0.83 \mathrm{Gyr}\right)$} \\
\hline$\Delta(B-R) / \Delta \log r$ & & & & & $t_{\text {age }}[\mathrm{Gyr}]$ & & & & \\
\hline-0.05 & 15.00 & 14.35 & 13.65 & 13.00 & 12.40 & 11.20 & 10.20 & 9.75 & 9.20 \\
\hline-0.07 & 15.00 & 14.00 & 13.10 & 12.20 & 10.60 & 9.75 & 9.00 & 8.60 & 7.80 \\
\hline-0.11 & 15.00 & 13.60 & 12.00 & 9.95 & 8.90 & 7.85 & 7.18 & 6.70 & 6.20 \\
\hline-0.13 & 15.00 & 13.35 & 11.10 & 9.35 & 8.35 & 7.20 & 6.65 & 6.10 & 5.63 \\
\hline
\end{tabular}

Note.- The second and third rows are the models constructed for the typical color gradients of $\Delta(B-R) / \Delta \log r=-0.09 \pm 0.02 \mathrm{mag} / \mathrm{dex}$. The models in the first and fourth rows are for $\Delta(B-R) / \Delta \log r=$ $-0.09 \pm 0.04 \mathrm{mag} / \mathrm{dex}$, within which gradients of almost all ellipticals in Peletier et al.(1990a) are included. 
Table 2. Properties of Model Galaxies at $z=0$

\begin{tabular}{|c|c|c|c|c|c|c|c|c|c|}
\hline $\log r / r_{\mathrm{e}}$ & -1 & -0.875 & -0.75 & -0.625 & -0.5 & -0.375 & -0.25 & -0.125 & 0 \\
\hline \multicolumn{10}{|c|}{ Metallicity Sequence $\left(t_{\text {age }}=15\right.$ Gyr $)$} \\
\hline \multirow[t]{4}{*}{$\log Z / Z_{\odot}$} & 0.254 & 0.240 & 0.219 & 0.201 & 0.182 & 0.168 & 0.142 & 0.123 & 0.101 \\
\hline & 0.254 & 0.230 & 0.204 & 0.177 & 0.153 & 0.123 & 0.092 & 0.066 & 0.034 \\
\hline & 0.254 & 0.216 & 0.177 & 0.136 & 0.084 & 0.034 & -0.002 & -0.046 & -0.080 \\
\hline & 0.254 & 0.210 & 0.158 & 0.101 & 0.056 & -0.002 & -0.046 & -0.118 & -0.140 \\
\hline \multirow[t]{4}{*}[\mathrm{Fe}/\mathrm{H}]{} & -0.069 & -0.088 & -0.112 & -0.131 & -0.151 & -0.165 & -0.192 & -0.213 & -0.236 \\
\hline & -0.069 & -0.100 & -0.128 & -0.155 & -0.181 & -0.213 & -0.245 & -0.273 & -0.307 \\
\hline & -0.069 & -0.115 & -0.155 & -0.199 & -0.254 & -0.307 & -0.346 & -0.394 & -0.431 \\
\hline & -0.069 & -0.121 & -0.175 & -0.236 & -0.284 & -0.346 & -0.394 & -0.474 & -0.497 \\
\hline \multirow[t]{4}{*}[\mathrm{Mg}/\mathrm{Fe}]{} & 0.467 & 0.473 & 0.475 & 0.475 & 0.476 & 0.477 & 0.478 & 0.479 & 0.481 \\
\hline & 0.467 & 0.474 & 0.475 & 0.476 & 0.478 & 0.479 & 0.481 & 0.483 & 0.486 \\
\hline & 0.467 & 0.475 & 0.476 & 0.479 & 0.482 & 0.486 & 0.489 & 0.493 & 0.496 \\
\hline & 0.467 & 0.475 & 0.477 & 0.481 & 0.484 & 0.489 & 0.493 & 0.500 & 0.503 \\
\hline \multirow[t]{4}{*}{$U-R$} & 2.166 & 2.158 & 2.144 & 2.132 & 2.118 & 2.109 & 2.089 & 2.076 & 2.060 \\
\hline & 2.166 & 2.152 & 2.134 & 2.115 & 2.098 & 2.076 & 2.055 & 2.036 & 2.014 \\
\hline & 2.166 & 2.143 & 2.115 & 2.085 & 2.049 & 2.014 & 1.988 & 1.957 & 1.933 \\
\hline & 2.166 & 2.139 & 2.101 & 2.060 & 2.029 & 1.988 & 1.957 & 1.904 & 1.888 \\
\hline \multirow[t]{4}{*}{$B-R$} & 1.632 & 1.627 & 1.620 & 1.614 & 1.608 & 1.604 & 1.595 & 1.589 & 1.583 \\
\hline & 1.632 & 1.624 & 1.615 & 1.606 & 1.599 & 1.589 & 1.580 & 1.572 & 1.563 \\
\hline & 1.632 & 1.619 & 1.606 & 1.593 & 1.578 & 1.563 & 1.552 & 1.539 & 1.528 \\
\hline & 1.632 & 1.617 & 1.600 & 1.583 & 1.569 & 1.552 & 1.539 & 1.516 & 1.509 \\
\hline \multirow[t]{4}{*}{$V_{606}-I_{814}$} & 0.576 & 0.574 & 0.572 & 0.569 & 0.567 & 0.565 & 0.562 & 0.559 & 0.556 \\
\hline & 0.576 & 0.573 & 0.570 & 0.566 & 0.563 & 0.559 & 0.555 & 0.552 & 0.548 \\
\hline & 0.576 & 0.571 & 0.566 & 0.561 & 0.554 & 0.548 & 0.543 & 0.536 & 0.531 \\
\hline & 0.576 & 0.570 & 0.564 & 0.556 & 0.550 & 0.543 & 0.536 & 0.525 & 0.522 \\
\hline
\end{tabular}


Table 2-Continued

\begin{tabular}{|c|c|c|c|c|c|c|c|c|c|}
\hline $\log r / r_{\mathrm{e}}$ & -1 & -0.875 & -0.75 & -0.625 & -0.5 & -0.375 & -0.25 & -0.125 & 0 \\
\hline \multicolumn{10}{|c|}{ Age Sequence $\left(t_{\mathrm{gw}}=0.83 \mathrm{Gyr}\right)$} \\
\hline \multirow[t]{4}{*}{$t_{\text {age }}$} & 15.00 & 14.35 & 13.65 & 13.00 & 12.40 & 11.20 & 10.20 & 9.75 & 9.20 \\
\hline & 15.00 & 14.00 & 13.10 & 12.20 & 10.60 & 9.75 & 9.00 & 8.60 & 7.80 \\
\hline & 15.00 & 13.60 & 12.00 & 9.95 & 8.90 & 7.85 & 7.18 & 6.70 & 6.20 \\
\hline & 15.00 & 13.35 & 11.10 & 9.35 & 8.35 & 7.20 & 6.65 & 6.10 & 5.63 \\
\hline \multirow[t]{4}{*}{$\log Z / Z_{\odot}$} & 0.254 & 0.254 & 0.254 & 0.254 & 0.254 & 0.254 & 0.254 & 0.254 & 0.254 \\
\hline & 0.254 & 0.254 & 0.254 & 0.254 & 0.254 & 0.254 & 0.254 & 0.254 & 0.253 \\
\hline & 0.254 & 0.254 & 0.254 & 0.254 & 0.254 & 0.253 & 0.253 & 0.253 & 0.253 \\
\hline & 0.254 & 0.254 & 0.254 & 0.254 & 0.253 & 0.253 & 0.253 & 0.253 & 0.253 \\
\hline \multirow[t]{4}{*}[\mathrm{Fe}/\mathrm{H}]{} & -0.069 & -0.069 & -0.069 & -0.070 & -0.070 & -0.070 & -0.070 & -0.070 & -0.070 \\
\hline & -0.069 & -0.069 & -0.070 & -0.070 & -0.070 & -0.070 & -0.070 & -0.070 & -0.070 \\
\hline & -0.069 & -0.069 & -0.070 & -0.070 & -0.070 & -0.070 & -0.070 & -0.070 & -0.070 \\
\hline & -0.069 & -0.070 & -0.070 & -0.070 & -0.070 & -0.070 & -0.070 & -0.070 & -0.070 \\
\hline \multirow[t]{4}{*}[\mathrm{Mg}/\mathrm{Fe}]{} & 0.467 & 0.467 & 0.467 & 0.467 & 0.467 & 0.467 & 0.467 & 0.467 & 0.467 \\
\hline & 0.467 & 0.467 & 0.467 & 0.467 & 0.467 & 0.467 & 0.467 & 0.467 & 0.467 \\
\hline & 0.467 & 0.467 & 0.467 & 0.467 & 0.467 & 0.467 & 0.467 & 0.467 & 0.467 \\
\hline & 0.467 & 0.467 & 0.467 & 0.467 & 0.467 & 0.467 & 0.467 & 0.467 & 0.467 \\
\hline \multirow[t]{4}{*}{$U-R$} & 2.167 & 2.154 & 2.140 & 2.126 & 2.114 & 2.099 & 2.082 & 2.068 & 2.054 \\
\hline & 2.167 & 2.147 & 2.129 & 2.112 & 2.089 & 2.068 & 2.047 & 2.031 & 2.012 \\
\hline & 2.167 & 2.139 & 2.110 & 2.074 & 2.043 & 2.013 & 1.988 & 1.963 & 1.938 \\
\hline & 2.167 & 2.133 & 2.098 & 2.058 & 2.023 & 1.989 & 1.961 & 1.932 & 1.904 \\
\hline \multirow[t]{4}{*}{$B-R$} & 1.633 & 1.627 & 1.621 & 1.614 & 1.609 & 1.603 & 1.596 & 1.590 & 1.584 \\
\hline & 1.633 & 1.624 & 1.615 & 1.608 & 1.599 & 1.590 & 1.581 & 1.574 & 1.566 \\
\hline & 1.633 & 1.620 & 1.607 & 1.593 & 1.579 & 1.567 & 1.553 & 1.539 & 1.525 \\
\hline & 1.633 & 1.618 & 1.603 & 1.586 & 1.570 & 1.553 & 1.538 & 1.522 & 1.506 \\
\hline \multirow[t]{2}{*}{$V_{606}-I_{814}$} & 0.577 & 0.575 & 0.573 & 0.570 & 0.569 & 0.566 & 0.564 & 0.561 & 0.560 \\
\hline & 0.577 & 0.574 & 0.571 & 0.569 & 0.565 & 0.561 & 0.559 & 0.558 & 0.563 \\
\hline
\end{tabular}


Table 2 - Continued

\begin{tabular}{lllllllllll}
\hline \hline $\log r / r_{\mathrm{e}}$ & -1 & -0.875 & -0.75 & -0.625 & -0.5 & -0.375 & -0.25 & -0.125 & 0 \\
\hline & & & & & & & & & & \\
& 0.577 & 0.573 & 0.568 & 0.562 & 0.559 & 0.562 & 0.557 & 0.547 & 0.537 \\
& 0.577 & 0.572 & 0.566 & 0.560 & 0.559 & 0.557 & 0.546 & 0.534 & 0.524 \\
\hline
\end{tabular}

Note.- The first, second, third and forth rows of each physical quantity correspond to those for the color gradient of $\Delta(B-R) / \Delta \log r=-0.05,-0.07,-0.11$, and $-0.13 \mathrm{mag} / \mathrm{dex}$, respectively. 
Table 3: Sample Elliptical Galaxies

\begin{tabular}{cccccccc}
\hline \hline $\mathrm{ID}^{\mathrm{a}}$ & $z^{\mathrm{b}}$ & $\begin{array}{c}I_{814^{\mathrm{c}}} \\
(\mathrm{mag})\end{array}$ & $\begin{array}{c}M_{F 450 W, A B}{ }^{\mathrm{c}} \\
(\mathrm{mag})\end{array}$ & $\begin{array}{c}\left(V_{606}-I_{814}\right)_{A B} \\
(\mathrm{mag})\end{array}$ & $\begin{array}{c}r_{\mathrm{e}} \\
(\mathrm{kpc})\end{array}$ & $\begin{array}{c}B / T^{\mathrm{d}} \\
\Delta\left(V_{606}-I_{814}\right)_{A B}{ }^{\mathrm{e}} \\
(\mathrm{mag})\end{array}$ \\
\hline $2-456.0^{\mathrm{f}}$ & 0.089 & $18.2^{\mathrm{g}}$ & $-19.20^{\mathrm{h}}$ & 0.610 & 1.4 & - & -0.02 \\
$4-241.1$ & 0.318 & 20.33 & -20.94 & 0.422 & 3.1 & 0.66 & - \\
$2-121.0$ & 0.475 & 20.03 & -20.94 & 1.071 & 3.5 & 0.87 & 0.10 \\
$3-790.0$ & 0.562 & 20.93 & -21.92 & 1.152 & 2.8 & 0.63 & 0.21 \\
$3-321.0$ & 0.677 & 20.92 & -22.17 & 1.490 & 7.0 & 0.81 & 0.09 \\
$4-744.0$ & 0.764 & 20.41 & -23.12 & 1.609 & 5.1 & 0.79 & 0.06 \\
$4-493.0$ & 0.847 & 21.17 & -22.89 & 1.670 & 4.0 & 0.81 & 0.02 \\
$2-251.0$ & 0.960 & 20.86 & -23.65 & 1.055 & 7.9 & 1.00 & - \\
$4-752.0$ & 1.013 & 20.88 & -24.10 & 1.855 & 6.9 & 0.87 & -0.14 \\
$4-928.0$ & 1.015 & 21.75 & -23.03 & 1.347 & 3.7 & 0.81 & -0.4 \\
\hline \hline
\end{tabular}

${ }^{a}$ Williams et al. (1996).

${ }^{b}$ Cohen et al. (1996).

${ }^{c}$ Abraham et al. (1999). We converted the $M_{F 450 W, A B}$ magnitudes into those in the adopted cosmology.

${ }^{d}$ Bulge-to-total luminosity ratio in the $I_{814}$-band taken from Marleau \& Simard (1998).

${ }^{e}$ Shifted zero-points to compare the model color gradients for typical elliptical galaxies with observed ones (see $\S 4)$.

${ }^{f}$ The $B / T$ ratio of this object is not in Marleau \& Simard (1998). However, we add it to our sample because its spectrum (from the Hawaii active catalog; Cowie 1997) as well as morphology show this galaxy is clearly a typical E or S0.

${ }^{g}$ Bouwens, Broadhurst, \& Silk (1998).

${ }^{h} b_{J}$-band absolute magnitude calculated by Bouwens et al. (1998). We also converted the magnitude into that in the adopted cosmology. 


\section{Figure Caption}

Fig. $1-V_{606}-I_{814}$ colors of our sample galaxies in a $10 \mathrm{kpc}$ aperture as a function of redshift. Solid line shows a color track for a passively evolving elliptical and dotted line for a non-evolving elliptical. Filled circles indicate red galaxies whose colors are nearly consistent with that of the passively evolving or non-evolving ellipticals. Open circles indicate the remaining bluer galaxies.

Fig. 2 - Azimuthally averaged radial surface brightness profiles of our sample galaxies in the $I_{814}$-band. (a) Red galaxies of which colors are consistent with that of a passively evolving galaxy. (b) Galaxies of which colors are bluer than that of a passively evolving galaxy (see also Figure 1). Filled circles show the data points and solid lines show the fitted lines with the $r^{1 / 4}$ law.

Fig. $3-V_{606}-I_{814}$ color maps for (a) the red galaxies whose colors are consistent with that of a passively evolving galaxy, and (b) the "blue" ellipticals. Plus sign of each object

denotes the centroid of the galaxy in the $I_{814}$-band image. The circle represents an effective radius centered on the centroid. Object ID, redshift, and effective radius are also shown. Note that a color bar is different from galaxy to galaxy, to show the color distribution clearly.

Fig. 4 - Observed color gradients of the red elliptical galaxies together with the model gradients by the age gradient. Filled circles represent the data points. Crosses refer to the data points which are not used in deriving slopes of color profiles. The models show the predicted color gradients seen at each object's redshift. Solid lines correspond to $\Delta(B-R) / \Delta \log \left(r / \mathrm{r}_{\mathrm{e}}\right)=-0.09 \pm 0.02 \mathrm{mag} / \mathrm{dex}$ at $z=0$, and the dotted lines correspond to $\Delta(B-R) / \Delta \log \left(r / \mathrm{r}_{\mathrm{e}}\right)=-0.09 \pm 0.04 \mathrm{mag} /$ dex at $z=0$. Zero-points of the observed colors are slightly shifted by $\Delta\left(V_{606}-I_{814}\right)$ in Table 3 to compare the observed gradients with the models. 
Fig. 5 - Same as Fig. 4, but for the case of the metallicity gradient.

Fig. $6-V_{606}-I_{814}$ color gradient versus redshift for our red sample galaxies. Filled circles represent color gradients of the sample ellipticals, which are determined within effective radii without the innermost regions of the galaxies. Solid and dotted lines indicate the evolutions of color gradients caused by the metallicity gradient and the age gradient, respectively. (See text for the model.)

Fig. 7 - Color gradients of the galaxies which have relatively blue colors. The color gradient of 4-928.0, which has a rather steep color gradient, is plotted together with a model color gradient made by age gradient fitted to the observed one by tuning ages of stellar populations in the model galaxy. (See text for the model.) The observed color for 4-928.0 is shifted by 0.4 mag to compare the model gradients. 


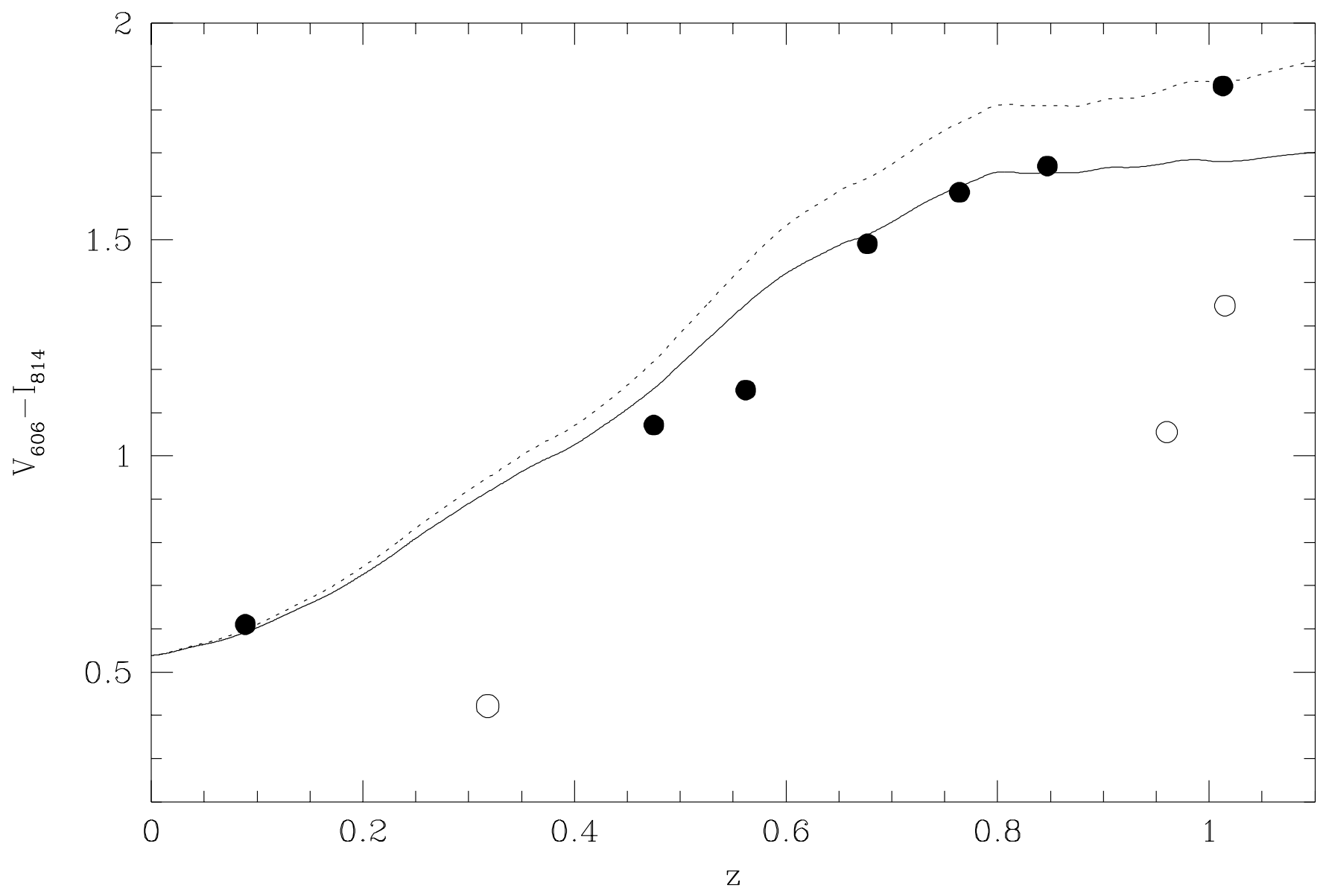



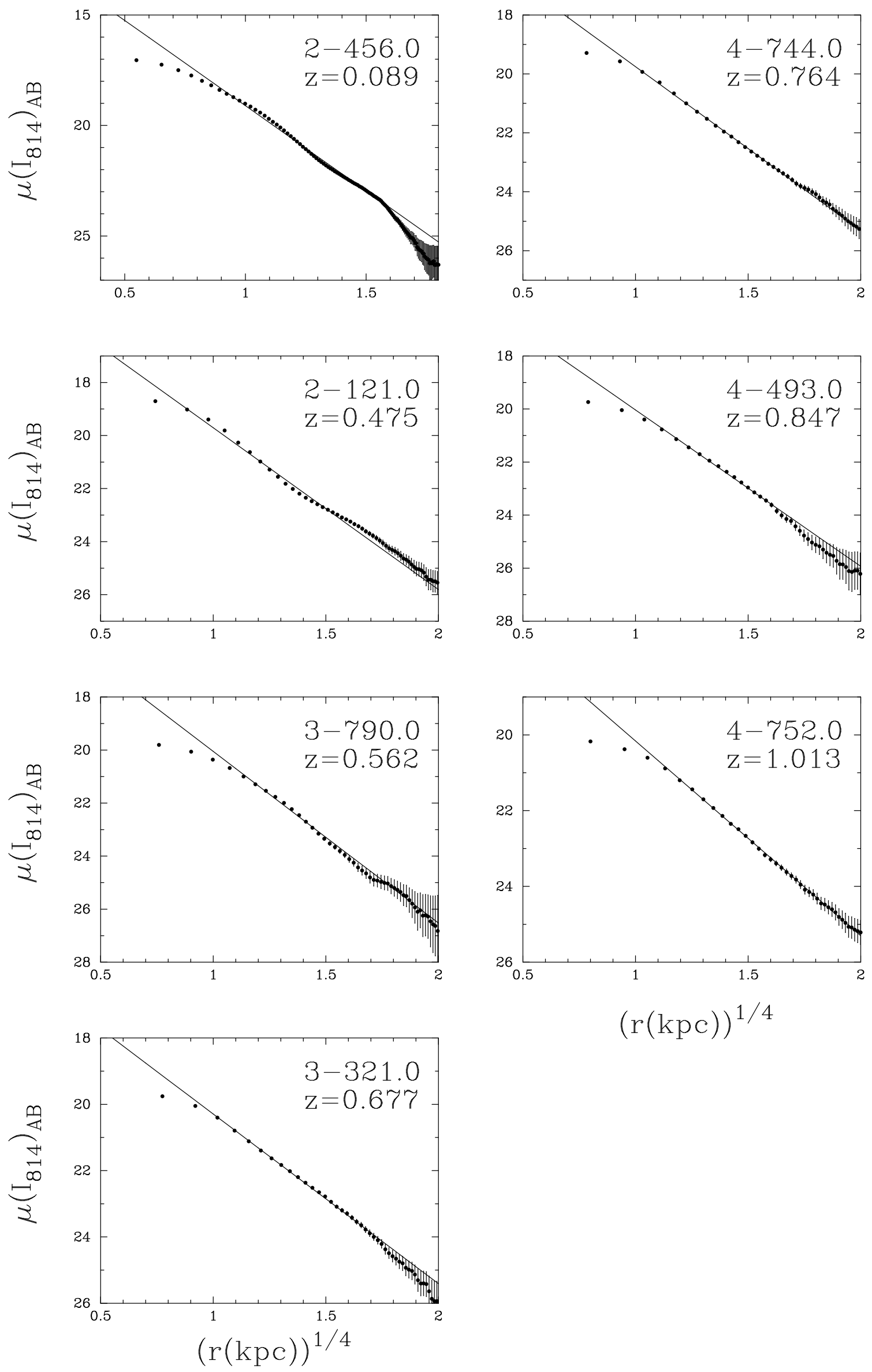

Figure 2(a) 

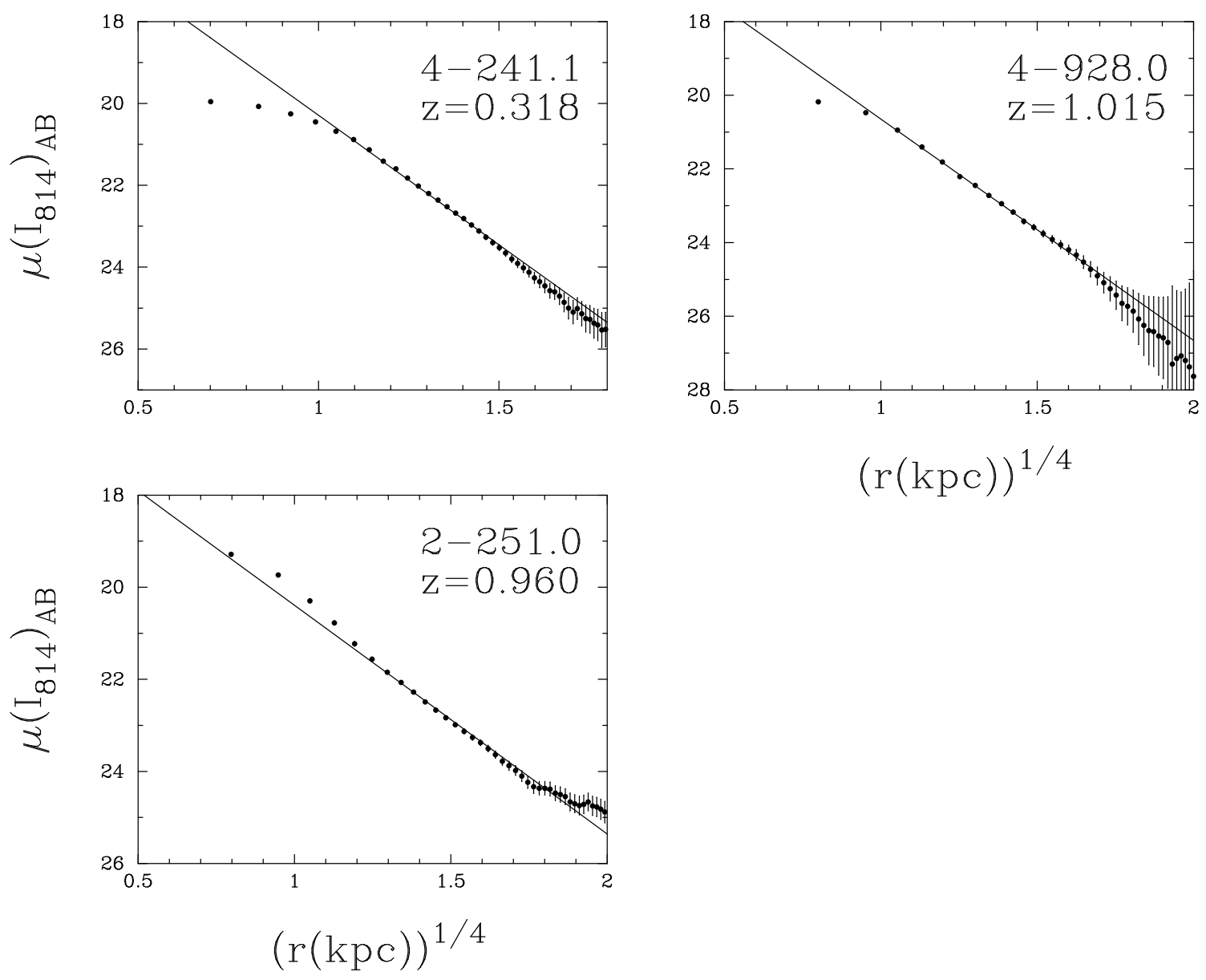

Figure 2(b) 

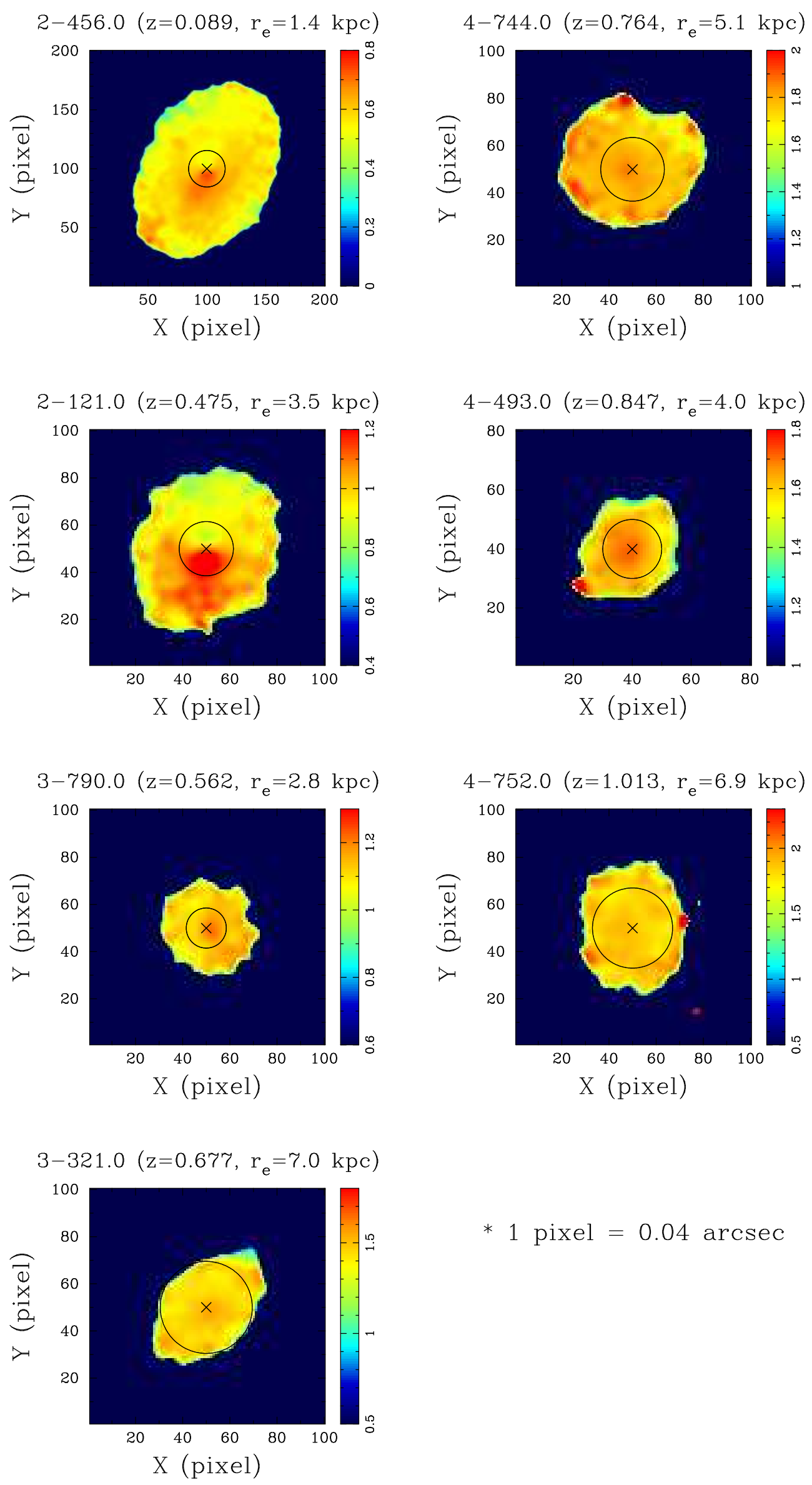

$* 1$ pixel $=0.04$ arcsec

Figure 3(a) 

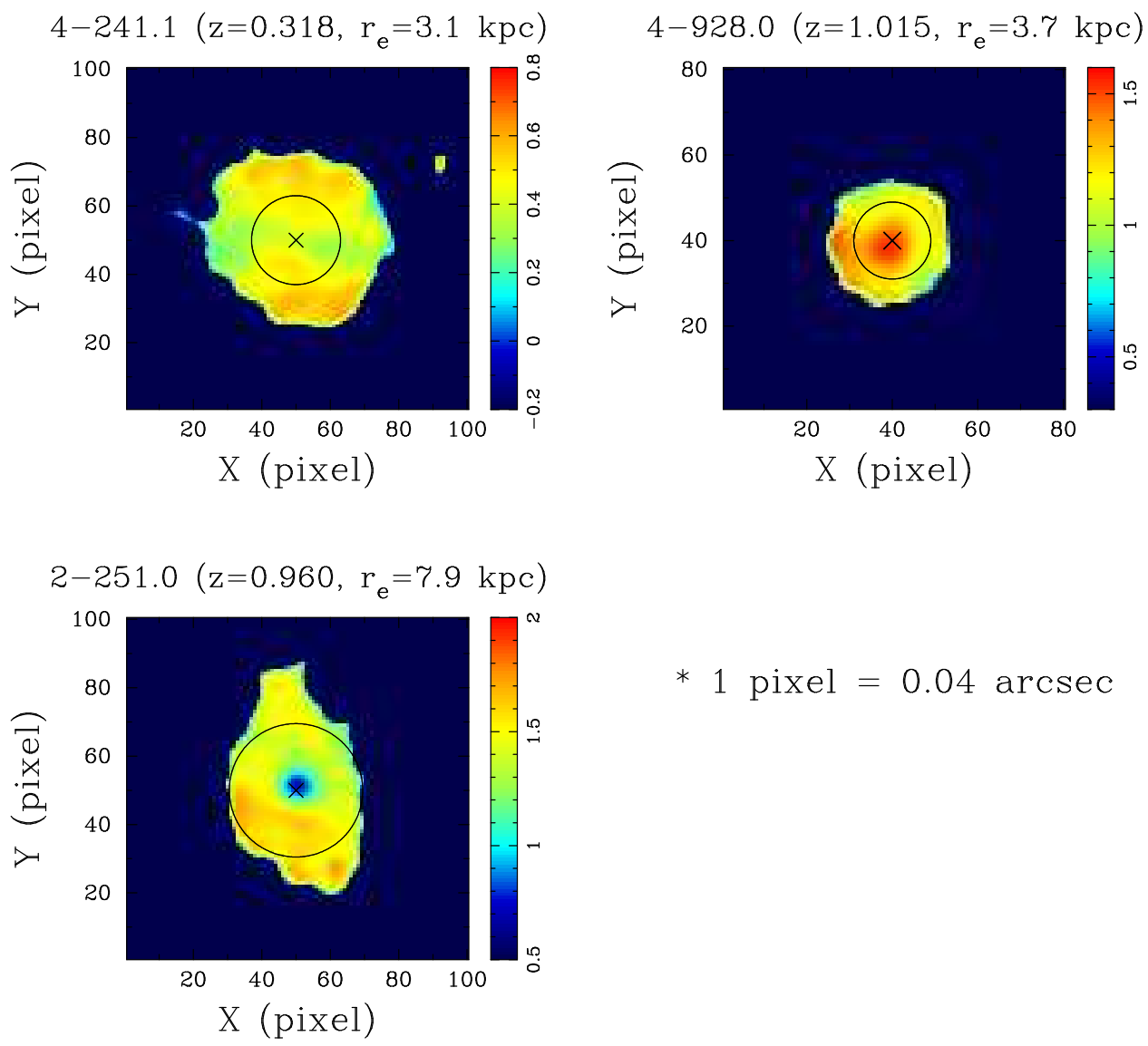

* 1 pixel $=0.04$ arcsec

Figure 3(b) 
Age Sequence
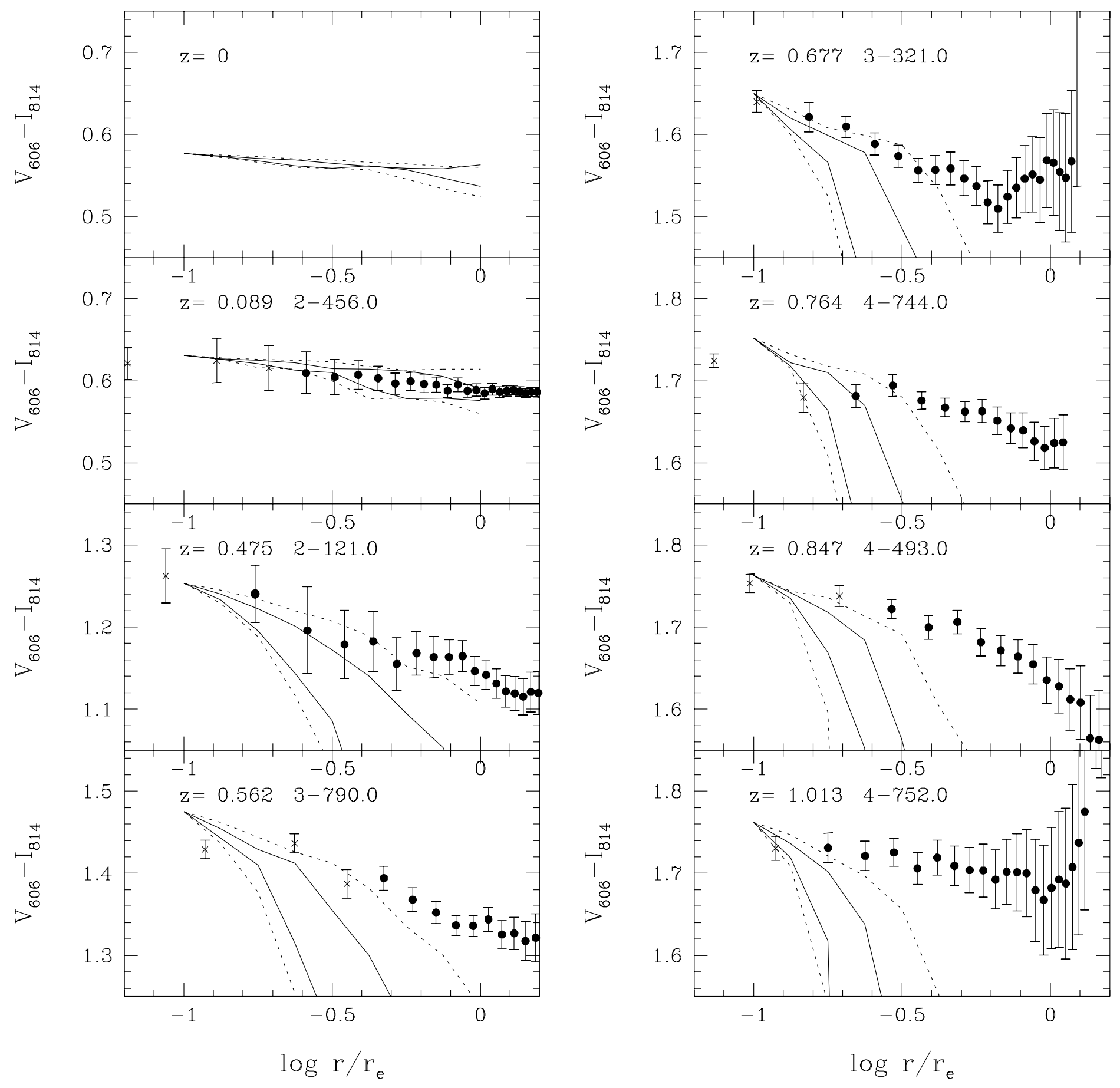
Metallicity Sequence
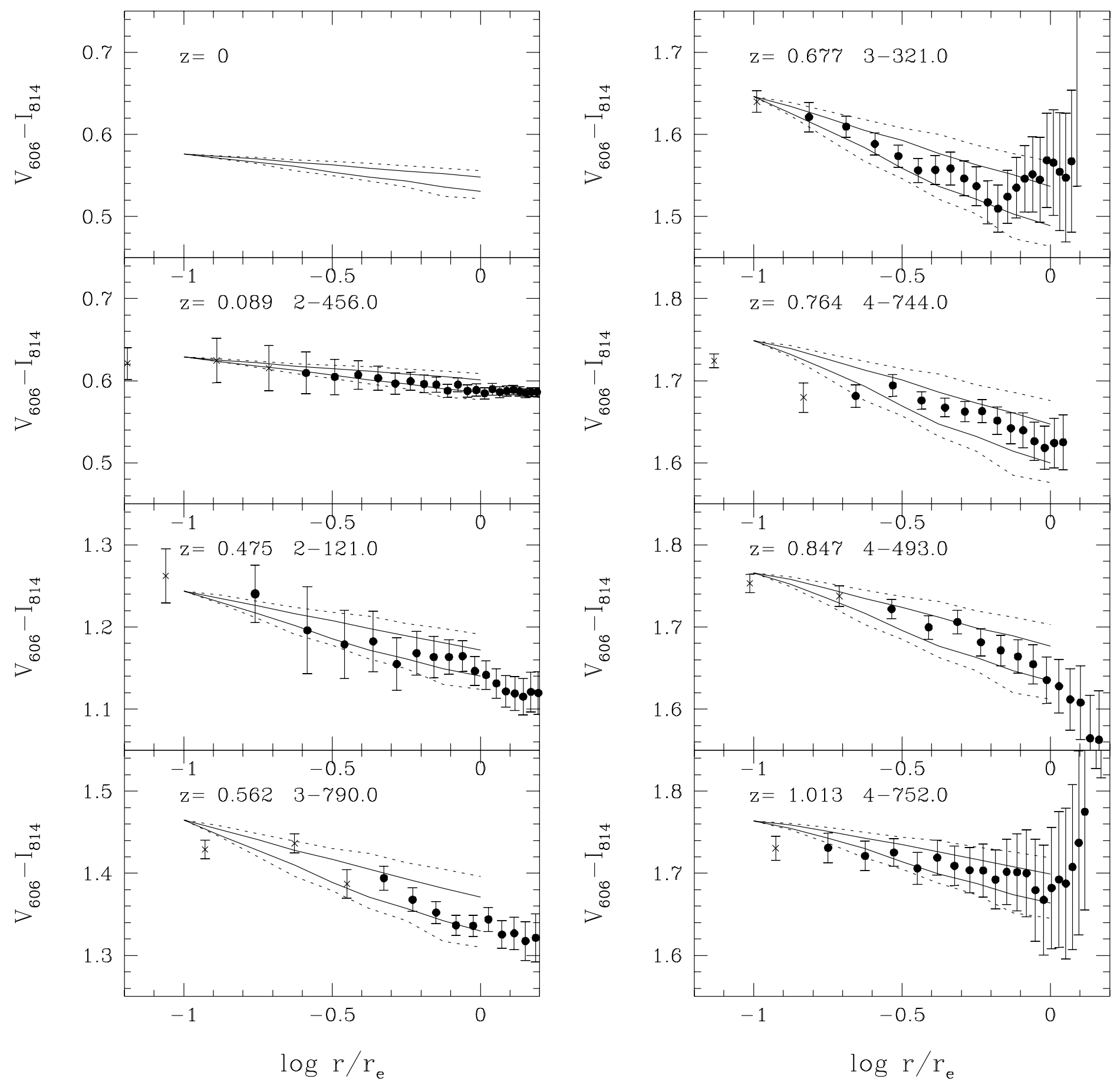


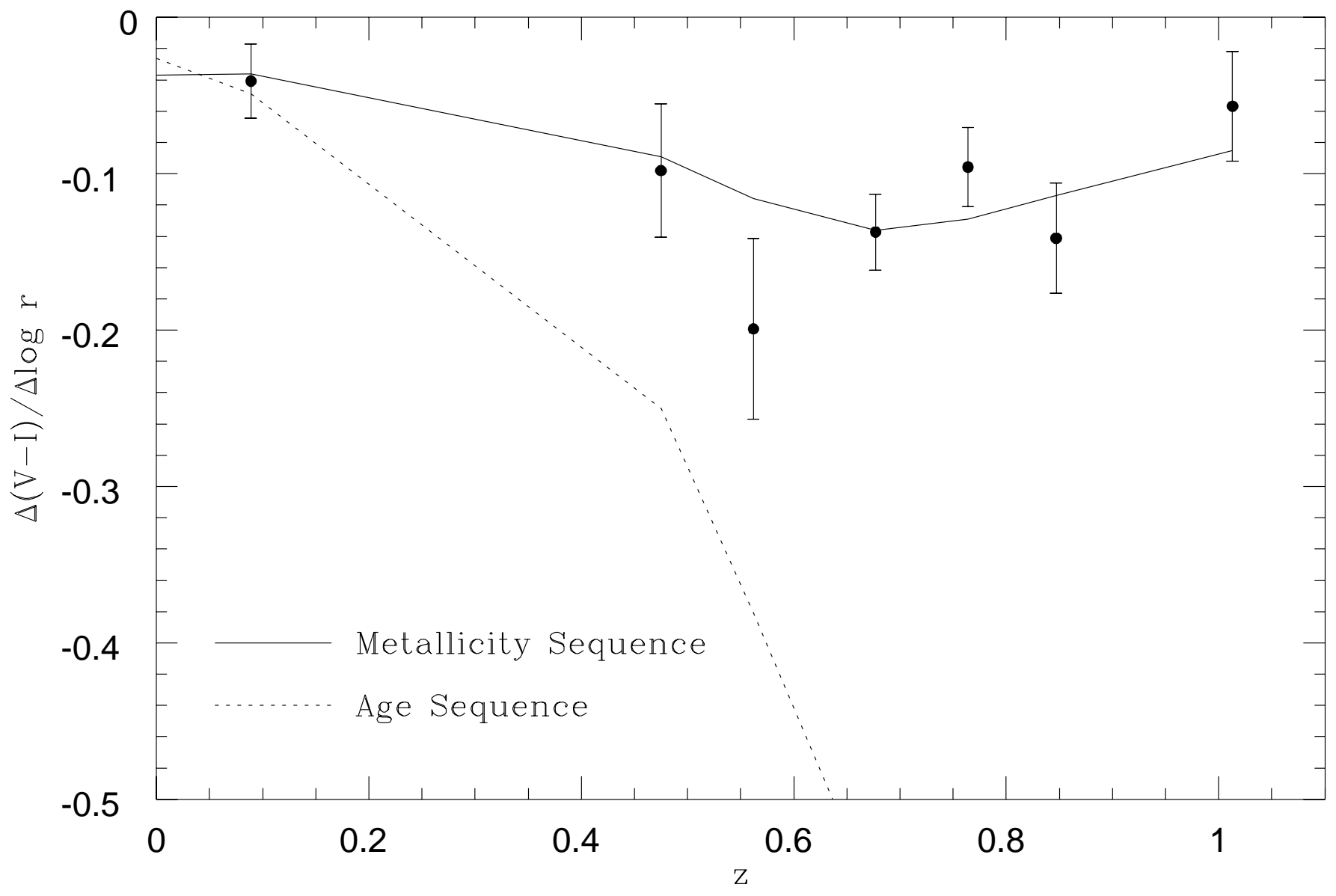




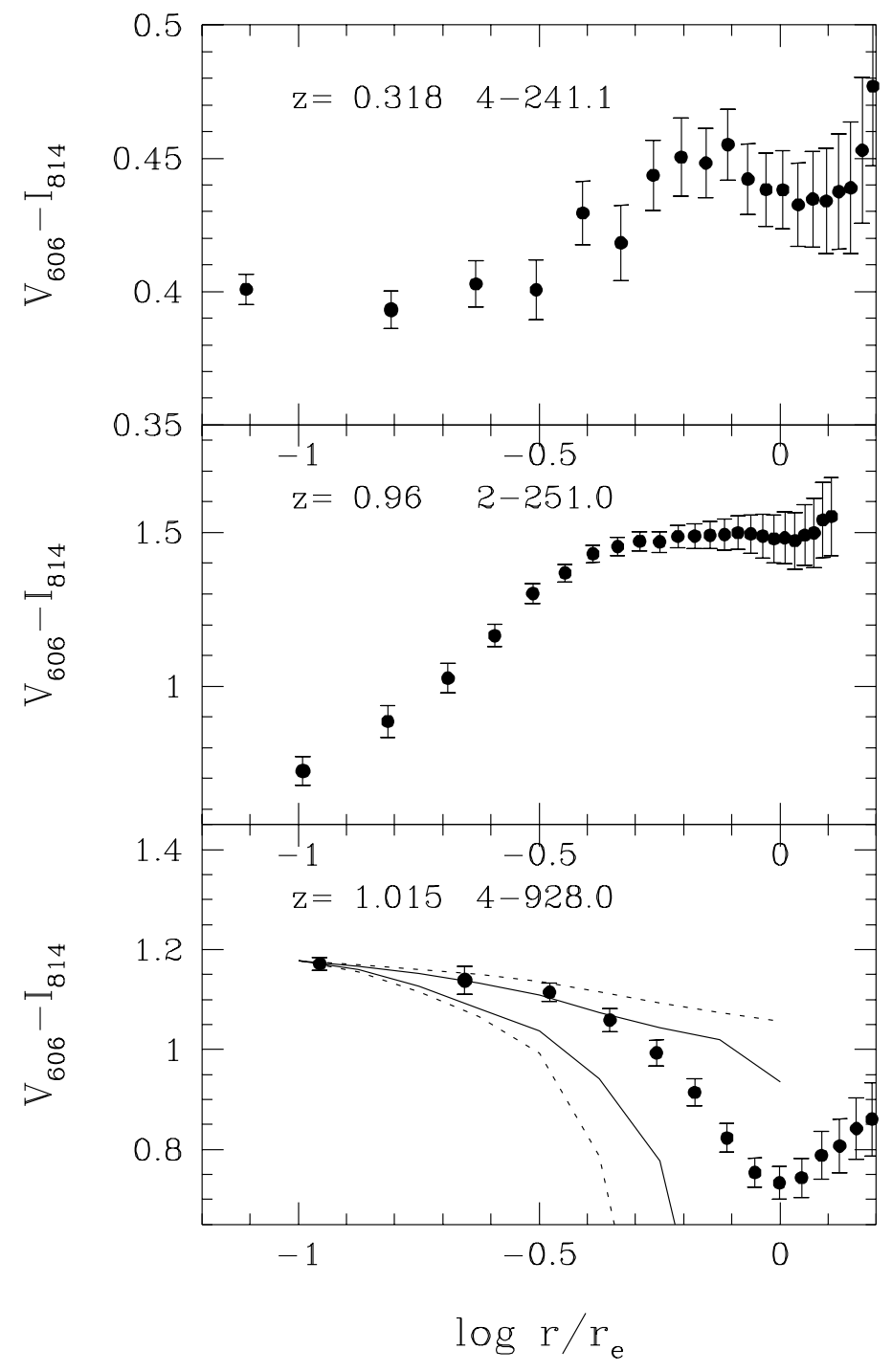

